\title{
Visual Communication Design of Intangible Cultural Heritage Based on Internet+
}

\author{
HUANG Wei-bo, ZENG Rou-mei, XIANG Han-dun, LI Xiao-dan, LIANG Wen-xin, LAI Ge-ling \\ Guangdong University of Foreign Studies, Guangzhou, China
}

\begin{abstract}
Wuchuan Piaose Art is a traditional folk cultural activity of the Han nationality in Wuchuan City, Guangdong Province. It is a comprehensive plastic art integrating drama, music, art, magic, acrobatics, and mechanics. Exploring and recording the intangible cultural heritage of Wuchuan in an Internet + and computer-aided way is of great significance to the practice and historical research of Wuchuan. It provides a folklore material for the artistic creation of figure paintings, which is of pioneering significance.
\end{abstract}

Keywords: Internet + , Piaose, intangible cultural heritage, visual communication design

\section{Introduction}

As an intangible cultural heritage, Piaose has a long history of development. This folk art has gained a lot of compliment and recognition. Nevertheless, in this time of the high-speed development of information technology, it is facing a great problem. Nowadays Internet+ platform provides a new way that adds new forms on visual communication design on it, which may promote its development (Cozzani, Pozzi, Dagnino, Katos, \& Katsouli, 2017).

\section{Development of Intangible Cultural Heritage}

\section{The History of Piaose}

As a folk art, Piaose has a long history which can date back to the Late Ming and Early Qing Dynasty. The name of Piaose means floating and coloring. Floating shows its condition which leaves the ground in the most of the performance time. Coloring has two parlance. One is the colorful plank named Seban that the actors stand. The another is the gorgeous and dressed up characters. Piaose is a folk blessing activity in the past. The actors standing on the Seban act the folk tales.

\footnotetext{
Acknowledgement: This study was financially supported by the Characteristic Innovation Projects, the Teacher-Student Co-research Category of School-Level Scientific Research Project of Guangdong University of Foreign Studies in 2018 (Grant No. 18SS14).

HUANG Wei-bo, Master, Senior Experimentalist, Education Technology Center, Experimental Teaching Center, Guangdong University of Foreign Studies, Guangzhou, China.

ZENG Rou-mei, undergraduate, School of Economics and Trade, Guangdong University of Foreign Studies, Guangzhou, China.

XIANG Han-dun, undergraduate, School of Art, Guangdong University of Foreign Studies, Guangzhou, China.

LI Xiao-dan, undergraduate, School of English Education, Guangdong University of Foreign Studies, Guangzhou, China.

LIANG Wen-xin, postgraduate student, School of Accounting, Guangdong University of Foreign Studies, Guangzhou, China.

LAI Ge-ling, School of Journalism and Communication, Guangdong University of Foreign Studies, Guangzhou, China.
} 
Piaose is related to the Pageant on Immortals formerly. People paraded it in the festival time. Now vehicles with dynamic replace the manual carts of the past. At the present stage a Seban can accommodate many people on it.

The most wonderful part of Piaose is that everything is supported by a stalk named "Segeng", which is hard to watch. The miracle is based on the principle of mechanics. Also, audiences on each side can see different three-dimensional scenes, which are magnificent and grand.

\section{The Audiences of Piaose}

Piaose is an art protected by country as a intangible cultural heritage. There are plenty of associations in the area they performance (Alivizatou-Barakou et al., 2017). The actors and researchers can often communicate with each other from place to place. From these thing, so to speak, they across the country work together. At the same time, the high artistic value also attracts attention from other places. A lot of people often ask the Piaose group to go performing, which also is the source of performers' income.

However, despite the importance attached to this art, not many people are willing to learn and inherit it. One of the brilliant scene of Piaose is the performance of child actors. Those who can stand on the Seban are need to choose from the clever, lively, and active boys and girls. It is difficult to select now. One of the reasons is that parents are not particularly willing to accept the inheritance of this folk art. So the situation that singles out performers is in a jam.

Meanwhile, Piaose's audiences are many fellow countrymen who are interested on it, or the groups of strangers who need Piaose to liven things up. Essentially, after many people have seen it, they are amazed by the miraculous of the Piaose and do not feel much about it.

\section{Visual Communication of Piaose}

The model of Piaose comes from drama; the drama can be said to be one of the most splendid pearls of Chinese culture. It covers the cultural customs of the dynasties, carries the pride and move of the Chinese nation. Wuchuan Piaose is based on this. In the conceiving process, the artist of Piaose embodies the aesthetic emotion and literary consciousness of his own time into the understanding of objects. Then the specific visual expressions are conveyed to certain targets in another special way. The adaptation of music, art, acrobatics, and styling makes it possible to display artistic emotions preferably in a small space. This also shows that the emotional concept has already penetrated into the visual design of Piaose. The traditional symbols are conveyed to the audience with different performances, satisfying people's spiritual and material needs. The design of works is integrated into emotions that the audience yearns, and attracts the audience with visual emotion. Thinking of the continuous uplifting influence of the opera culture of all generations on people, the design is rather reasonable. The art of Piaose is showed in the form of street parade. The spatial distance is different; the actions of the actors are various. With the development of science and technology, automatic devices such as smoke, water spray, and fire-breathing are added into the performance, thus the blend of vision is created. People through the eyes receive more diverse information and the individual's visual thinking processing is not the same.

This shows that in the same performance, with the differences of viewing angle, height and distance, the acceptance of visual time, each audience can obtain different impressions. This is the creativity of visual perception that Wuchuan Piaose brings to people. 


\section{Obstacles and Problems in the Inheriting Process of Piaose}

Over the past 400 years, Piaose has experienced various obstacles and gradually developed. However, in the inheriting process, it still face great problems. If we use functionalism to define it, it successfully meets people’s cultural needs as a non-material culture and belongs to the category of spiritual civilization. From the cultural point of view, when a culture begins to lose its function and influence, it will begin to disappear by degrees (Zhang, 2018).

In other words, in the process of inheritance, if the connotation and cultural significance it conveys can not be accepted by people with the development of the times, it will go downhill like many intangible cultures that can not be spread, and may eventually be forgotten by people (Wang \& Liu, 2015).

\section{Visual Communication Design in "Internet+"}

\section{Traditional Visual Communication Design}

Visual communication design is a process that takes a certain purpose as the guide, conveys some specific information to the target through visual art form, and influences the target. In the field of art design, visual communication design exists as one of its forms (Wang \& Liu, 2015).

Traditional visual design refers to the use of words, graphics, and color to convey things in the new media era. It mainly uses illustration, font art, and label signs to represent specific things. There are four steps in the process of "who", "what to do", "to whom to convey", and "the effect to convey". In the traditional visual communication, Piaose performers deduce this art and convey it intuitively to the audience. The effect is both on-site and temporary visual memory. In fact, as early as in ancient times, people used visual communication design in all aspects. The treasures that remain in the long river of history have been waiting for modern people to use more scientific research methods to explore the constitution and reasons of its principles (Mcnely, 2013).

\section{New Progress and Influences of Visual Communication Design in "Internet+"}

In the age of Big Data, visual communication has entered a new stage. In addition to paper media, the use of multimedia has begun to fill social life. In the information age of fragmentation, such as Wechat, Web Pages, and applications, the media of packaging information is upgraded, which makes information disseminated more widely and deeply. In the Internet+ era, the media has been updated, and visual communication design has also undergone new changes (Thorlacius, 2009).

Emotional design is a process that not only reflects emotions, but also pays more attention to its expression of artistic sensibility. By incorporating design elements into the emotional design, it forms an interactive process of injecting emotional ideas into its design works. The emotional concept that emerged in the design process began with a people-oriented purpose: The design of the work carries the comfort of the human civilization, and center in people. Emotionalization of the work can inprove the communication between the work and the human being, and increase the aesthetics of the human-centered perspective. In this way, the resonance of human emotion can be aroused and more added value can be brought to the work.

\section{Advantages of New Visual Communication in the Age of Big Data}

In the visual communication, Piaose was mostly conveyed by the performers to the audience formerly. It is difficult for ordinary people to understand the complex connotation of the introduction of Piaose through paper 
media. With traditional visual communication design, it is hard to fully display the charming of it, too. As a non-material cultural heritage, Piaose has complex show and exquisite performance. And it requires a large amount of information to convey.

Under the big data mode, the huge information network has made a great contribution to restoring the beauty of Piaose. As we all know, in the Information Age, the media can not be affected by a large number of factors, and transmit complex and diverse information as far as possible. Similarly, information has been free from geographical constraints, and people all over the world can experience and feel the charm of new visual communication.

Therefore, under the background of "Internet+", visual communication design can play a great role in the inheritance of Piaose.

\section{Visual Communication Design of Piaose With the Aid of "Internet+"}

\section{Film Recording Resource Library}

For how to spread Piaose more widely, it is more efficient to let the audience intuitively feel the charm than to let the audience know what the Piaose is. Therefore, the film recording resource library is a good new way of visual communication. There are many columns in the big data platform of intangible cultural heritage, but the information is not complete enough. And it is necessary to create a separate resource bank for Piaose film records.

Mobile applications or computer programs can be chosen to create a resource library. In terms of communication, computer programs can accommodate more data. But it's hard to get more people to download the plug-in substantially. Therefore, mobile APP is more acceptable to the public.

In APP, it is easy to make full use of the advantages of the big data era and put the Piaose introduction into it. And it can be divided into different types according to the different regions. Also, it can insert the viewing of Piaose's video of different ages according to the time sequence. Meanwhile, the existing intangible cultural heritage video data can be collated, patched, format transformed, and retrieved and abstracted. Moreover, many long videos can be edited into small videos of wonderful scenes. Because of it, visual communication can achieve the best effect. The benefit of doing so is convenient for people to popularize, study, and research.

Last but not least, the resource library should be constantly updated. Also, forums and comments need to be opened. People from all walks of life can make suggestions on how to make the Piaose visual communication more popular. After integrating a large number of data, the film materials can also highlight the artistic value of Piaose to the greatest extent.

\section{Visual Design of Web Pages}

Web visual design can also make this folk art have a better inheritance. Starting with the establishment of links to web sites, the pictures and videos of intangible cultural heritage can be designed. And they can be presented on the homepage. The overall style of the web page should take into account both uniqueness and unity. Also, the artistic effect is one of the most important part. For example, the logo design can connect the sign with the region. Flash animation can be used to simply simulate the process of Piaose performance by prettifying pictures and words.

The stereoscopic effect and versatility of Piaose need modern visual design to achieve, that is, 3D modeling. Piaose can be rendered in 2D for 3D or modeled directly to present the whole performance at 360 degrees and 
show it on the web page. In this way, audiences can experience the details of the performance in various levels and aspects through rotating perspective. At the same time, VR technology can also be arranged in web design. Stronger visual effect can make Piaose more touching.

\section{Conclusion}

The combination of intangible cultural heritage and visual communication design is a new mode of inheritance and development in the Internet+ era. As a intangible cultural heritage, how to use this new design to spread is also a problem that needs to be studied at present. Through the discussion of modern visual communication design, traditional culture can also have a new way of expression, a new pair of wings.

\section{References}

Alivizatou-Barakou, M., Kitsikidis, A., Tsalakanidou, F., Dimitropoulos, K., Giannis, C., Nikolopoulos, S., ... Grammalidis, N. (2017). Intangible cultural heritage and new technologies: Challenges and opportunities for cultural preservation and development. In Mixed reality and gamification for cultural heritage (pp. 129-158). Cham: Springer.

Cozzani, G., Pozzi, F., Dagnino, F. M., Katos, A. V., \& Katsouli, E. F. (2017). Innovative technologies for intangible cultural heritage education and preservation: The case of i-treasures. Personal \& Ubiquitous Computing, 21(2), 1-13.

Mcnely, B. J. (2013). Visual research methods and communication design. In ACM International Conference on Design of Communication (pp. 123-132). Greenville, North Carolina, USA.

Rees, H. (2017). Intangible cultural heritage in contemporary China: The participation of local communities. International Journal of Heritage Studies, 24(5), 1-3.

Thorlacius, L. (2009). Visual communication in web design—analyzing visual communication in web design. In International handbook of internet research (pp. 455-476). Dordrecht: Springer.

Wang, J. H., \& Liu, L. (2015). Research on computer graphics design and visual communication design. Applied Mechanics \& Materials, 713-715, 2191-2194.

Zhang, J. (2018). Intangible cultural heritage and self-healing mechanism in Chinese culture. Folklore Studies, 76(2), 197-226. 